\title{
Thrombin Effects on Cultured Nerve Cells: Clinical Implications and Evidence for a Novel Mechanism of Neuronal Activation ${ }^{a}$
}

\author{
R. MICHAEL SNIDER ${ }^{b}$ \\ Neuroscience Lab \\ Mental Health Research Institute \\ University of Michigan \\ Ann Arbor, Michigan 48104-1687
}

\begin{abstract}
Central nervous system tissue in vivo is composed of a wide heterogeneity of cell types, such as neurons, glia, and vascular endothelium. One experimental approach that is commonly used to circumvent the inherent problems encountered in studying neuronal phenomena is the use of cloned nerve cells such as have been derived from the $\mathrm{C}$ 1300 murine neuroblastoma. One clone, designated N1E-115, has been extensively characterized both biochemically and electrophysiologically, and represents a widely used cell culture system for neurobiological studies.' Inasmuch as cloned cells constitute a population of neurons derived from a single cell, specific questions can be addressed to a single aspect of neuronal function.
\end{abstract}

\section{Neurotransmitter Receptor-Mediated Cyclic GMP Synthesis}

Clone N1E-115 cells have been shown to possess functional muscarinic and histamine $H_{1}$ receptors that, when activated, cause a rapid and marked increase in the intracellular concentration of cyclic guanosine monophosphate (GMP) ${ }^{2-4}$ The molecular hypothesis for receptor-mediated cyclic GMP formation that researchers have held for the past several years involves calcium channel activation as the effector that couples receptor activation to cyclic GMP formation. ${ }^{5}$ This hypothesis results primarily from the observations that extracellular calcium ions $\left(\mathrm{Ca}^{2+}\right)$ are necessary for receptor responses, and that $\mathrm{Ca}^{2+}$ ionophores (such as A23187 or X537A) can cause cyclic GMP formation.

In the course of investigating the molecular mechanism of neuronal activation, we gathered direct evidence indicating that a large $\mathrm{Ca}^{2+}$ influx did not occur as a result of neurotransmitter agonist stimulation. ${ }^{6}$ Instead, results were obtained suggesting that neurotransmitter agonists may be coupled to guanylate cyclase (the enzyme that synthesizes cyclic GMP from guanosine triphosphate (GTP) by involvement of phospholipase $A_{2}$ and the release of arachidonic acid metabolites. ${ }^{6}$ Phospholipase $A_{2}$ is the

${ }^{a}$ This work was supported by USPHS Grant NS 20920.

${ }^{b}$ Present address: T Cell Sciences, Inc., 840 Memorial Dr., Cambridge, MA 02139. 
enzyme that catalyzes the release of arachidonic acid from the $R_{2}$ position of membrane phospholipids. Thrombin is a serine protease and is the central regulatory enzyme in hemostasis. Among the major biological effects of thrombin in causing coagulation of blood are cleavage of fibrinopeptide $\mathbf{A}$ to convert fibrinogen into clotable fibrin and stimulation of platelet degranulation and aggregation. Moreover, thrombin stimulation of platelets is known to result in the activation of phospholipase $A_{2}$ and cause the release of arachidonic acid. ${ }^{11,12}$

\section{Thrombin Effects on Neuroblastoma Cells}

To explore further the possible involvement of phospholipase $A_{2}$ and arachidonic acid release in neurotransmitter action, we tested the ability of thrombin to stimulate cyclic GMP formation in intact N1E-115 cells. Thrombin incubation with intact neuroblastoma cells caused a marked $\mathrm{Ca}^{2+}$-dependent elevation in cyclic GMP levels with a peak around 30-60 seconds. ${ }^{7}$ The time course and $\mathrm{Ca}^{2+}$ dependency of the thrombin-induced cyclic GMP response was virtually identical with other neurotransmitter agonists, such as carbachol and histamine, which stimulate cyclic GMP formations. ${ }^{2,4} \mathrm{The}^{\mathrm{E}} \mathrm{EC}_{50}$ (median effective concentration) for the thrombin response ranged from 0.08-0.44 NIH units $/ \mathrm{ml}$, which averaged 1.0-1.5 $\mathrm{nM}$, and the cyclic GMP levels increased by 4.1 to 19 -fold over basal levels measured in intact cells. ${ }^{7}$

We sought to establish that this effect was specific for thrombin and to determine if receptors for this protease existed on nerve cells. Trypsin, which is another serine protease, but with a broad specificity, was about 100 -fold less potent and less effective in stimulating the cyclic GMP response as compared with $\alpha$-thrombin. Moreover, thrombin treated with diisopropylphosphofluoridate, to inactivate the enzymatic site, was 300 - to 500 -fold less potent as compared with thrombin. ${ }^{8}$ Thus, it is likely that the enzymatic activity of thrombin is involved in the cyclic GMP response of neuroblastoma cells. Thrombin antagonists also were evaluated for effects in this experimental preparation. Hirudin, a specific thrombin antagonist isolated from the leech salivary gland, inhibited cyclic GMP formation in approximately 1:1 stoichiometry, and a competitive inhibitor of thrombin, termed DAPA (dansylarginine- $N$-(3-ethyl1,5-pentanediyl) amide) produced a shift to the right in the thrombin concentration cyclic GMP response and yielded a $K_{\mathrm{i}}$ value of $17 \mathrm{nM}^{7}$

Thrombin binding to intact neuroblastoma cells studied using [ $\left.{ }^{125} I\right]$ thrombin in a radioligand binding assay, are characterized as high-affinity receptors with a $K_{\mathrm{d}}$ (equilibrium dissociation constant) of $1.4 \mathrm{nM}$ that number approximately 30000 per cell. ${ }^{9}$ In addition, low-affinity binding with a very high capacity was also found. We believe that the high-affinity binding site $\left(K_{\mathrm{d}}=1.4 \mathrm{nM}\right)$ is the functional receptor coupled to the cyclic GMP response $\left(\mathrm{EC}_{30}=1.2 \mathrm{nM}\right)$. Moreover, $\left[{ }^{125} \mathrm{I}\right]$ thrombin also binds to human brain and spinal cord homogenates, ${ }^{10}$ suggesting that our findings in neuroblastoma cells are not artifactual but, in fact, may represent an aspect of normal or pathological neuronal physiology.

\section{Significance and Clinical Implications}

It is unlikely that under normal conditions blood-born clotting factors ever come into direct contact with nerve cells in the brain. The possibility exists, however, that one or more proteases with thrombin-like specificity might be synthesized by neurons, 
released, and cause activation of other neurons (i.e., a neuromodulatory function). A point of greater interest concerns the function that thrombin interaction may play in the neuropathology subsequent to cerebrovascular accidents, head trauma, or similar events in which the blood comes into direct contact with the brain.

Trauma to the central nervous system such as following cerebrovascular hemorrhage and concussive or projectile head injury clearly results in a compromised bloodbrain barrier. Consequently, nerve cells are directly exposed to blood-borne factors. The resultant in vivo situation is profoundly complex and, for that reason, it has been difficult to ask questions designed to provide discrete answers concerning the neuronal pathophysiology involved in the injury and healing process.

An extremely exciting possibility concerns whether thrombin may possess mitogenic activity in nerve cells. As stated above, little is known about the neuronal cellular pathophysiology following stroke or traumatic nervous system injury. If thrombin is capable of stimulating differentiated neurons, as we have shown, neurons surviving the initial traumatic event may be induced to restore functional synaptic contacts. These processes may, in part, underlie a regenerative function that occurs with time following a cerebrovascular accident.

\section{A New Hypothesis of Neurotransmitter Receptor-Mediated Cyclic GMP Formation}

The $\mathrm{Ca}^{2+}$ dependency and time course of thrombin's activation of neuroblastoma cells is similar to that of muscarinic or histaminergic stimulation. Moreover, the receptor-mediated biochemical effects of thrombin in causing platelet activation are well characterized and involve activation of phospholipase $\mathrm{A}_{2}$ and release of arachidonic acid. ${ }^{11,12}$ Interestingly, phospholipase $A_{2}$ activity is the rate-limiting step in the generation of free arachidonic acid, and it is known to be a highly $\mathrm{Ca}^{2+}$-dependent enzyme. Released arachidonic acid can undergo oxidative metabolism by way of the cyclooxygenase or lipoxygenase pathways to form the prostaglandins or the hydroxyeicosatetraynoic acids, respectively.

Pharmacologic studies were carried out to evaluate the involvement of phospholipase $A_{2}$ and arachidonic acid metabolites in cyclic GMP formation. Inhibition of phospholipase $\mathbf{A}_{2}$ with quinacrine resulted in a concentration-dependent attenuation of receptor-mediated cyclic GMP formation. Quinacrine was effective in blocking the response elicited by thrombin, carbachol, and histamine with nearly equal potency. ${ }^{6.8}$ This observation suggests that all the above agents that cause a receptor-mediated cyclic GMP response do so by the same mechanism. We conducted experiments to examine whether quinacrine inhibited the cyclic GMP response to receptor agonists in a nonspecific manner. Quinacrine did not inhibit the cyclic GMP response caused by sodium nitroprusside (a guanylate cyclase stimulant) at concentrations that totally blocked receptor responses.

Involvement of arachidonic acid metabolites in cyclic GMP formation was also investigated. Indomethacin, a drug that inhibits cyclooxygenase and formation of prostaglandins, did not inhibit the receptor-mediated response. ${ }^{6,8}$ In fact, in many experiments, indomethacin actually potentiated by $10-40 \%$ the cyclic GMP levels measured in response to agonist stimulation. Inhibition of the lipoxygenase pathway of arachidonate metabolism with nordihydroguaiaretic acid (NDGA) or eicosatetraynoic acid (ETYA) blocked receptor-mediated cyclic GMP synthesis in a concentrationdependent manner. As was observed with quinacrine, the responses evoked by throm- 
bin, carbachol, and histamine were affected similarly by NDGA or ETYA. We believe this observation also supports the hypothesis that neurotransmitter receptors couple to their respective cyclic GMP responses by a similar molecular mechanism.

Based on the observations detailed above, we have proposed a new hypothesis for the molecular mechanism of neurotransmitter receptor-mediated cyclic GMP formation. ${ }^{6}$ We believe this scheme of molecular events following receptor activation and leading to cyclic GMP formation is consistent with all of the experimental observations outlined above.

\section{REFERENCES}

1. Richelson, E. 1979. The use of cultured cells in neurobiological studies. Physiol. Pharmacol. Biochem. 26: 81-120.

2. Matsuzawa, H. \& M. NiRenBerg. 1975. Receptor-mediated shifts in cGMP and cAMP levels in neuroblastoma cells. Proc. Nat. Acad. Sci. USA 72: 3472-3476.

3. Richelson, E., F. G. Prendergast \& S. Divinetz-Romero. 1978. Muscarinic receptormediated cyclic GMP formation by cultured nerve cells-ionic dependence and effects of local anesthetics. Biochem. Pharmacol. 27: 2039-2048.

4. Richelson, E. 1978. Histamine $H_{1}$ receptor-mediated guanosine $3^{\prime}, 5^{\prime}$-monophosphate formation by cultured mouse neuroblastoma cells. Science 201: 69-71.

5. Richelson, E. \& E. El-FAKAHANY. 1981. The molecular basis of neurotransmission at the muscarinic receptor. Biochem. Pharmacol. 30: 2887-2991.

6. SNider, R. M., M. MCKinney, C. Forray \& E. Richelson. 1984. Neurotransmitter receptors mediate cyclic GMP formation by involvement of arachidonic acid and lipoxygenase. Proc. Nat. Acad. Sci. USA 81: 3905-3909.

7. SNIDER, R. M. \& E. Richelson. 1983. Thrombin stimulation of guanosine, 3',5'-monophosphate formation in murine neuroblastoma cells (clone N1E-115). Science 221: $566-568$.

8. Snider, R. M., M. McKinney, J. W. Fenton II \& E. Richelson. 1984. Activation of cyclic nucleotide formation in murine neuroblastoma N1E-115 cells by modified human thrombins. J. Biol. Chem. 259: 9078-9081.

9. SNIDER, R. M., M. MCKINNEY \& E. Richel.son. 1986. Thrombin binding and stimulation of cyclic guanosine monophosphate formation in neuroblastoma cells. Semin. Thromb. Hemostasis. 12: 253-262.

10. MCKInNeY, M., R. M. SNider \& E. Richeison. 1983. Thrombin binding to human brain and spinal cord. Mayo Clin. Proc. 58: 829-831.

11. Lapetina, E. G., M. M. Billah \& P. Cuatrecasas. 1981. The initial action of thrombin on platelets. J. Biol. Chem. 256: 5037-5040.

12. Billah, M. M. \& E. G. LAPETINA. 1982. Formation of lysophosphatidylinositol in platelets stimulated with thrombin or ionophore $A_{23187}$. J. Biol. Chem. 257: 5196-5200. 